\title{
Adaptive scheduling in deficit irrigation - a model-data fusion approach
}

\author{
M. M. Holloway-Phillips ${ }^{1}$, W. Peng ${ }^{2}$, D. Smith ${ }^{2}$ \& A. Terhorst ${ }^{2}$ \\ ${ }^{1}$ Tasmanian Institute of Agricultural Research, Australia \\ ${ }^{2}$ Tasmanian ICT Centre, Commonwealth Scientific and Industrial \\ Research Organisation, Australia
}

\begin{abstract}
The technological demands required to successfully practice either targeted irrigation control and/or deficit irrigation strategies are currently reliant on numerical models which are often underutilised due to their complexity and low operational focus. A simple and practical real-time control system is proposed using a model-data fusion approach, which integrates information from soil water representation models and heterogeneous sensor data sources. The system uses real-time soil moisture measurements provided by an in situ sensor network to generate site-specific soil water retention curves. This information is then used to predict the rate of soil drying. The decision to irrigate is made when soil water content drops below a pre-defined threshold and when the probability of rainfall is low. A deficit strategy can be incorporated by lowering the irrigation "refill" point and setting the fill amount to a proportion of field capacity. Computer simulations show how significant water savings can be achieved through improved utilisation of rainfall water by plants, spatially targeted irrigation application, and precision timing through adaptive control
\end{abstract}

Keywords: deficit irrigation, wireless sensor network, adaptive irrigation scheduling, model-data fusion, irrigation decision tree.

\section{Introduction}

Australia is facing a severe water shortage due to below-average rainfall received over the past decade. The agricultural sector is the hardest hit by this as irrigation accounts for almost $65 \%$ of total water use nationally [1]. Long-term climate forecasts suggest that this situation is unlikely to improve [2]. Therefore 
the agricultural industry must become increasingly innovatively in its efforts to use water more effectively. Two techniques which have been successfully used to improve water use efficiency include improved irrigation scheduling practices [3] and the reduction of water application rates using deficit irrigation strategies [4].

Optimising the timing of irrigation events involves being able to sense when the soil water reaches a threshold level estimated either directly using moisture sensors, or indirectly through meteorological data (for example evapotranspiration - ET). Incorporation of short-term weather forecasts can improve the projected need for irrigation and save water by increasing the amount of rainfall utilised by plants [5]. Solving spatial and temporal variation in soil water dynamics, however, becomes more demanding requiring real-time monitoring capabilities (e.g. soil moisture sensors, hydrologic models and remote sensing), and/or a high degree of empirical soil physical data, both of which can be very expensive and labour intensive.

Where water is particularly limiting, further reductions in irrigation water will be required. This has been addressed through the concept of deficit irrigation. Deficit irrigation strategies deliberately allow crops to sustain some degree of water deficit and sometimes an associated yield reduction through a significant reduction of irrigation water. The classic deficit irrigation strategy involves supplying water at levels below full ET throughout the season. In practice this has commonly been achieved by either irrigating at the same frequency but applying less water during each irrigation event, or maintaining the amount of water per irrigation but increasing the interval between irrigations [6]. Where water accounting has been used, irrigation decisions have generally been based on the 'trigger level' concept of available soil water.

The technological demands required to successfully practice either targeted irrigation control and/or deficit irrigation strategies are currently reliant on numerical models which are often underutilised due to their complexity and low operational focus [7]. A simple and practical real-time control system is proposed using a model-data fusion approach, which integrates information from soil water representation models and heterogeneous data sources to improve output resolution and irrigation decision making.

In comparison to other numerical models which act more in a simulation capacity for testing various water-management/allocation strategies at the basin level, the model presented here is to be applied as a functional monitoring tool for automated control at the paddock level. It has the advantage that it can adapt the irrigation plans in real-time to meet the desired soil water conditions defined by the irrigation rules. Model accuracy increases with observation as the model learns soil water flow relationships. Whilst this initial training period may result in a degree of inaccuracy, this is outweighed by the fact that the model can learn soil physical attributes in situ. Assigning sensors to similar soil spatial zones through an initial training phase removes some of the high labour and expense demands associated with sensor technology. The low manual inputs needed to use this technology and the limited knowledge required to interpret the output are expected to improve uptake by the broader irrigation community. 
This paper presents the model-data fusion approach to irrigation control and how the technology may be applied in practising deficit irrigation. Whilst field validation has not yet been undertaken, preliminary simulation results suggest that water savings can be achieved through consideration of spatial variation and short-term weather forecasts into the irrigation decision-making process.

\section{Model data fusion in deficit irrigation}

Sensor network technologies are proving to be powerful tools for monitoring real-time changes in the environment. There is a need to aggregate complex data from heterogeneous sensors, providing a rich, multidimensional picture of the system. Much work has been done in the field of data fusion [8-11], which deals with the functional transformation of data into human apprehensible information. Studies looking at "situation awareness" focus on developing models based on how humans perceive and comprehend their environment and subsequently anticipate potential change $[12,13]$. Situation is defined as a set of environmental conditions and system states with which the participant is interacting, and can be characterised by a set of information, knowledge, and response options [14]. Situation Awareness consists of three levels of mental models [12]:

- Perception is the process by which a participant identifies the status, attributes, and dynamics of relevant elements in the environment.

- Comprehension is concerned with prioritising and evaluating the information obtained from perception according to their relevance to current goals;

- Projection deals with forecasting future states of elements in the environment based on the awareness achieved in Perception and Comprehension.

To provide decision support in the real-time environment, a situation-aware information fusion system must handle problems in association with inflexible knowledge representation. With respect to the soil hydrologic environment, such information includes the spatial-temporal distribution and evolution of soil moisture which are non-deterministic due to complex interactions among environmental factors like soil profile, precipitation, ET, etc. This requires a system with the capability to learn new knowledge and adapt to the environment. What follows is a description of the model-data fusion approach used to develop a simple real-time irrigation control system.

\subsection{A framework for model-data fusion}

Model-data fusion is the approach via which an information system learns environmental behavioural models and utilises the learned models to better represent and predict environmental phenomena. Figure 1 shows the framework of a model-data fusion system. The framework consists of Sensors, Effectors, a Classifier, a Model Learner, and a Situation Projector; an Environment Representation Model which represents soil characteristics, and a Decision 
Model which captures a set of irrigation decisions in relation to environmental variables.

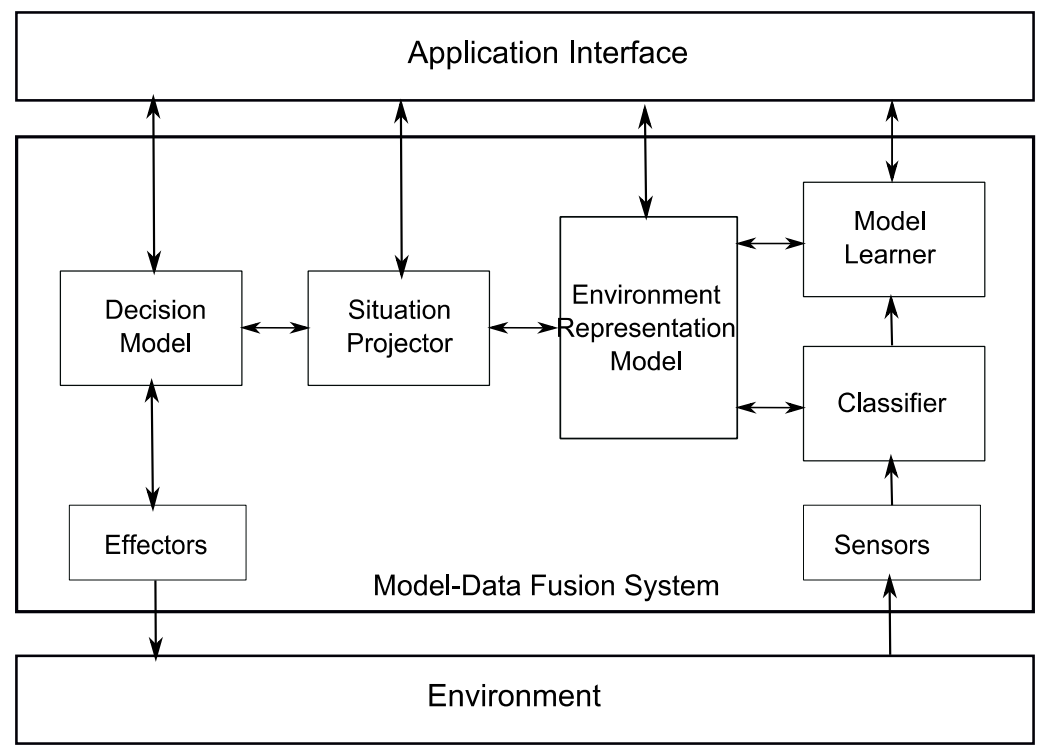

Figure 1: The framework for a situation-aware model-data fusion system.

Sensors are data collectors, which continuously observe the environment. During the learning stage, the Model Learner learns a Representation Model of the environment. The Representation Model captures empirical relationships between data inputs and outputs. The learnt model is then used by the Classifier to identify environmental properties in a perception-related process. The Situation Projector "comprehends" a situation by prioritising information according to how it impacts on the current goal. It also generates a projection (or anticipation) based on the Environment Representation Model. A Decision Model contains strategies to fulfil the goal under various circumstances. Effectors perform tasks which are specified in a decision. The application interface enables a user to view information generated at different components so as to make decisions (or choose a recommendation). Interactions between model and sensed data ground the decision making process in a dynamic environment.

\subsection{Learning the environment representation model via in situ sensors}

Soil moisture, rainfall, surface run-off, wind, humidity, temperature are some of the important types of data that can improve our understanding of the environment in the context of irrigation. The sensors adopted in this research are soil volumetric moisture sensors, soil water potential sensors (gypsum blocks), soil temperature sensors and an automatic weather station. 
The Environment Representation Model, is a description of environmental variables and their interrelationships, and is used for predicting future conditions. The environment is represented as three dimensional soil cubes with each cube representing a finite element of soil behaviour. One aspect of soil behaviour of significant importance is the relationship between volumetric water content $(\theta)$ and water potential $(\psi)$. This relationship forms what is known as the soil moisture retention curve, and is commonly used to estimate the water-holding capacity of soil horizons within the root-zone.

Within each cube, a gypsum block measures soil water potential. Correlated water potential measurements between spatially neighbouring cubes enables localised clusters to be formed. Each cluster of cubes with similar water potential behaviour can be assigned a volumetric soil moisture sensor. This approach reduces the number of expensive volumetric sensors that need to be employed within the field. For each cube, the relationship between water potential and volumetric water content is then learnt by the Model Learner during a training phase where sensor data is collected from the field. This scenario is shown in Figure 2.

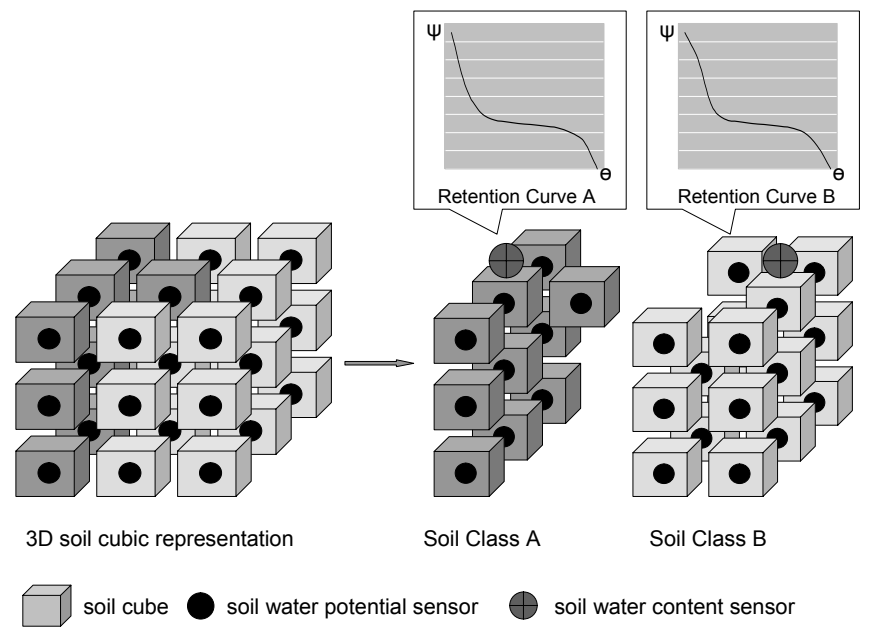

Figure 2: $\quad$ A 3D cubic representation of soil and classification based on water retention characteristics.

Direct measurement of retention curves in the field, particularly at such fine scale, should provide considerable improvement to the understanding of soil behaviour compared with indirect, coarser approximations, such as the use of reference soil types, which fail to take into account soil heterogeneity within the field. Reference data may also be inaccurate due to its measurement in a different environment (i.e. the laboratory) and under different conditions. There is considerable discrepancy between the retention curve of a particular soil type, measured in both the laboratory and the field [15]. 
Another common approach to obtain the retention curve is to use Pedotransfer (PTF) functions. PTF functions predict the water retention curve from using basic, easily measured properties of the soil. Consequently, PTF can be very crude approximations and are often only optimised for particular soil types and conditions.

Ideally, the training of retention curves within the field should cover measurements over a significant proportion of the retention curve (i.e. from saturation to the permanent wilting point), including the dynamic hysteresis within wetting and drying periods. However, it is unlikely to be possible to measure the complete range of potential and volumetric observations in situ during the training phrase. For unmeasured regions, the curve can be predicted by using non-linear regression or neural network methods such as the Mualemvan Genutchen model [16], to fit the measured data to retention curve models until the relevant conditions have been observed.

Retention curves are unique for different soil types as they are dependent upon the physical properties of the soil, such as porosity and particle size. Therefore deriving retention curves in situ also has the advantage of providing a coarse taxonomy of the soil composition of each cube, thus considering spatial variability in irrigation decisions. This can be achieved by fitting a model to reference and field retention curves and then comparing their shape parameters.

\subsection{Predicting soil water content}

Predicting how the soil water content changes over time becomes an important parameter for deciding when to irrigate. The high frequency of data being collected from field sensors offers the potential to predict the short term future of soil water content and enables irrigation plans to continually adapt to meet the target objectives.

One of the challenges in predicting changes in soil water content is that wetting and drying behaviour is a non-deterministic process which is dependent upon a number of environmental attributes. Hence, prediction of the soil water content through modelling is complex, as demonstrated by the Hydrus program [17]. Hydrus has not yet been proven to be an applicable solution to support real time decision making due to the complexity in the physical modelling.

We assume that the rate of soil water change can be learnt from previous soil water behaviour in the field. A relationship between the rate of soil water content change and the other dependent variables such as precipitation and ET can be learnt through observation. The benefit of this approach is that the system is not attempting to learn the physical model itself, but instead the relationship between soil water change rate and other dependent system attributes. In a sense, it is equivalent to the real time learning of PTF functions from the field.

With the learned characteristics of each soil class, the Classifier can locate environmental variables in the Representation Model (retention curves) and the Situation Projector can subsequently infer the needs for irrigation by calculating the distance to the refill point $\left(\theta_{\text {threshold }}\right)$ for each soil class. Figure 3 illustrates this concept. 


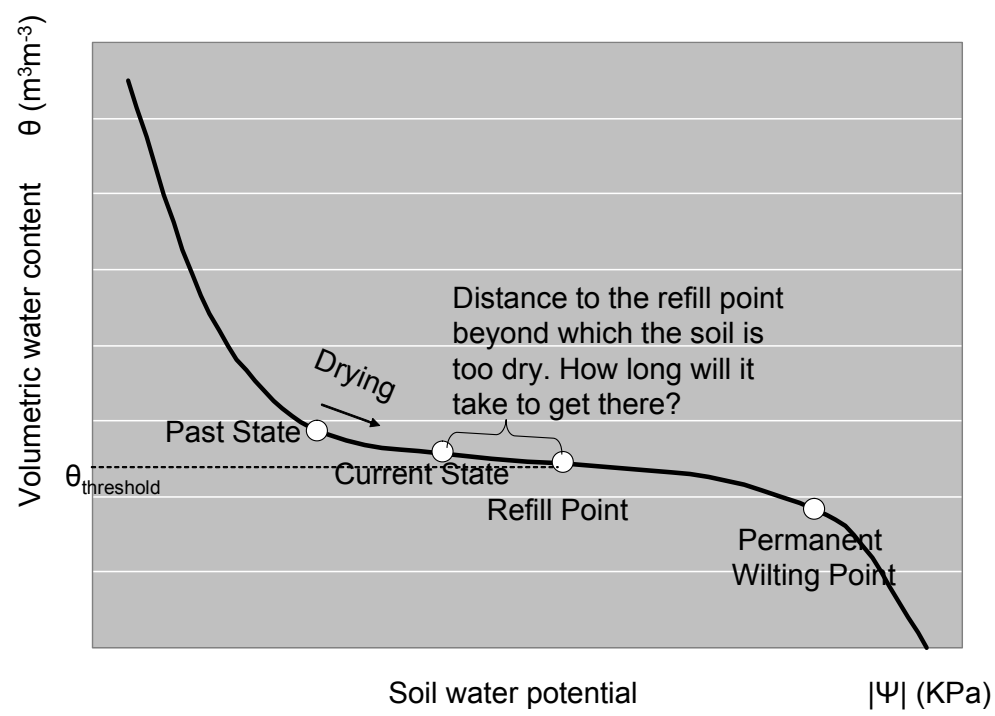

Figure 3: Predicting the rate of soil drying based on field-derived soil moisture retention curves, for irrigation scheduling decisions.

\subsection{Technical-driven deficit irrigation decision model}

Besides the soil drying status, the decision of whether to irrigate and the amount of water to apply at the current time, is dependent upon other information including rain forecasts, ET demand, current water allocation and other plant related factors. Decision tree learning using inductive inference has been the method adopted for deciding when to irrigate. Inductive learning methods identify features that empirically distinguish positive from negative observed training examples. Figure 4 presents the results obtained from a decision tree learning algorithm. Each non-leaf node stands for a test on an attribute. Edges of the decision tree coming from the nodes are values of attributes for that node. Leaf nodes are used to represent design decisions for selecting a deficit ratio. Numbers in parentheses illustrate an observation for the class defined in the leaf node. For example, "10.0" indicates that there are ten positive observations and no negative observations for that class.

The decision rules encoded within the model consider historical irrigation treatments, and generalises empirical knowledge that can be applied to select an appropriate irrigation treatment for a specific combination of field conditions. Three environment variables form the decision tree. These variables describe whether a soil cube is at the refill threshold point (node "reachRefillPoint"), if it is currently raining (node "currentRain"), and the probability of expected rain in 12 hours (node "forecastRain"). Leaf nodes illustrate irrigation treatments. Irrigation strategies are obtained by traversing from root node to a leaf node. For example, some interesting rules can be found: 


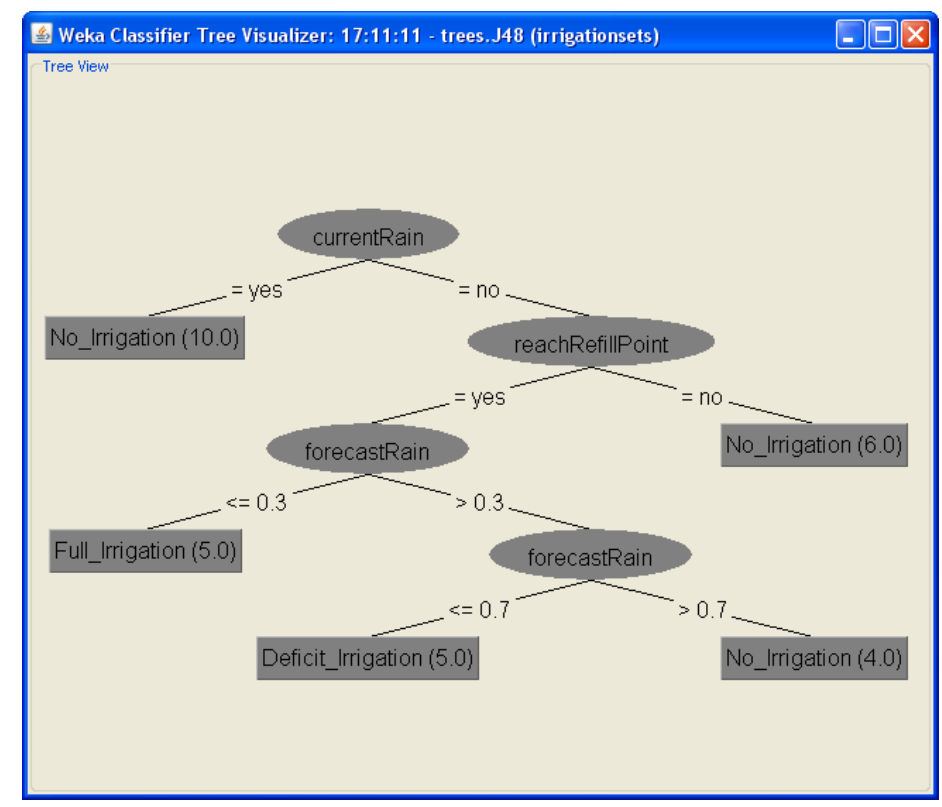

Figure 4: A decision tree structure learnt from running a C4.5 [18] algorithm from WEKA [19] on 46 instances of deficit irrigation treatments.

- if the soil cube has reached refill point whilst it is not raining, and the probability of the predicted rainfall is greater than 0.7 , irrigation water should not be applied. Irrigation is postponed until the next decision time, for example, in 4 hours;

- if the soil cube has reached refill point whilst it is not raining, and the probability of the predicted rainfall is between $0.3-0.7$, deficit irrigation should be applied to mitigate the risks of water stress on plant growth. The pre-defined deficit ratio is applied to the accumulated ET since the last irrigation or rainfall event to calculate the irrigation requirements.

Information fused from various sensors can be used to construct a recommendation. The system can also learn and evolve the decision model in real time. For example, by monitoring the plant response (e.g. remotely sensed canopy temperature), if the pre-defined plant-based thresholds have not been met under the applied irrigation deficit ratio, the system can re-adjust to a lower ratio when similar environmental conditions prevail. If required, the irrigation decision model can also act autonomously to schedule irrigation events.

\section{Model simulation}

An irrigation simulator was developed in NetLogo [20] to demonstrate how the model works and contributes to water use efficiency when taking account of rainfall information. Scenarios of a simulated deficit irrigation model and a point sensor-triggered irrigation system are discussed. 
The irrigation simulator uses field data including ET and rain gathered from Elliot research farm (North West Tasmania; $41^{\circ} 06^{\prime} \mathrm{S}, 145^{\circ} 46^{\prime} \mathrm{E}$ ) during the period of 2 November to 21 December, 2007. This data is scaled down to fit into the simulation model with essential variances unchanged. The simulator consists of two agent-based models:

1. A deficit irrigation model, which integrates local weather conditions and rainfall forecasts to decide when and how much to irrigate, and targeted irrigation control, with application to only those areas that have reached the refill threshold point;

2. A point sensor-based irrigation model, which only uses threshold soil moisture to trigger irrigation with uniformed application.

To exemplify the effect of different irrigation treatments on water usage, both models run under the same sets of environmental conditions including ET, rainfall, soil profile characteristics, and boundary conditions such as irrigation rate and drainage rate. A 3D soil water diffusion model is used to simulate soil water content changes under these conditions. The soil is represented by $3 \mathrm{D}$ cubes, as shown in Figure 3. The simulated soil consists of loam, clay and sand at different horizons (Figure 5). The soil diffusion model is a simplified view of the tendency of water to move from one soil cube to another, based on differences in soil water contents and soil porosities. Considering a soil cube and its neighbouring cubes, soil water diffusion can be simulated by recalculating the distribution soil water between cubes:

$$
T_{i}=P_{i} \times \frac{\sum_{1}^{n} T}{\sum_{1}^{n} P}-E T-D R
$$

where $T_{i}$ denotes soil water content unit for cube $i$

$i$ is the cube for recalculation

$n$ is the number of neighbours for cube $i$

$P$ is the porosity of soil cube $i$

$E T$ stands for water loss from evapotranspiration for cube $i$ (only top level cubes have ET)

$D R$ denotes water unit loss from drainage for cube $i$ over boundary (only the bottom-level cubes consider DR)

Eqn. (1) describes how soil water diffusion is calculated in the simulator. Environmental changes can cause the soil water content to re-distribute. Figure 6 also shows an example of how to apply Equation 1 to calculate simulated soil water content in cube $i$. By iterating all soil cubes, the effect of environmental conditions can be represented through soil water diffusion.

Point sensor-based irrigation treatments apply water uniformly to the whole paddock. Rainfall is affected by the same environmental conditions as irrigation. Water is lost through drainage and ET with some water units retained in the soil. Figure 6 shows the accumulated irrigation water usage during 50 time units of an irrigation treatment with a model initialised to pre-defined soil water contents.

In this scenario, 6170 units of water have been consumed as a result of 21 irrigation events. 


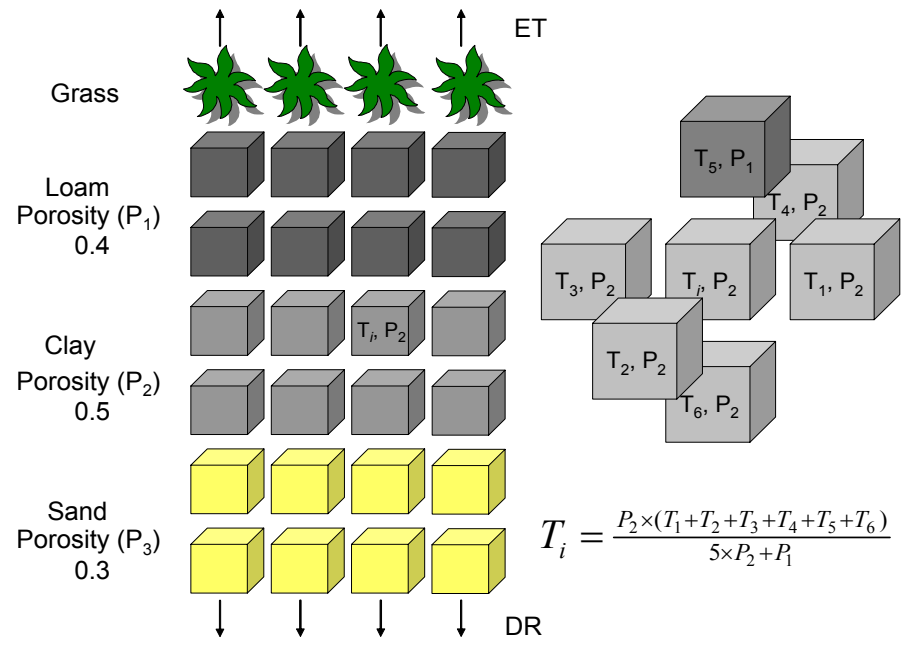

Figure 5: Soil profiles and the diffusion approach used in the simulator.

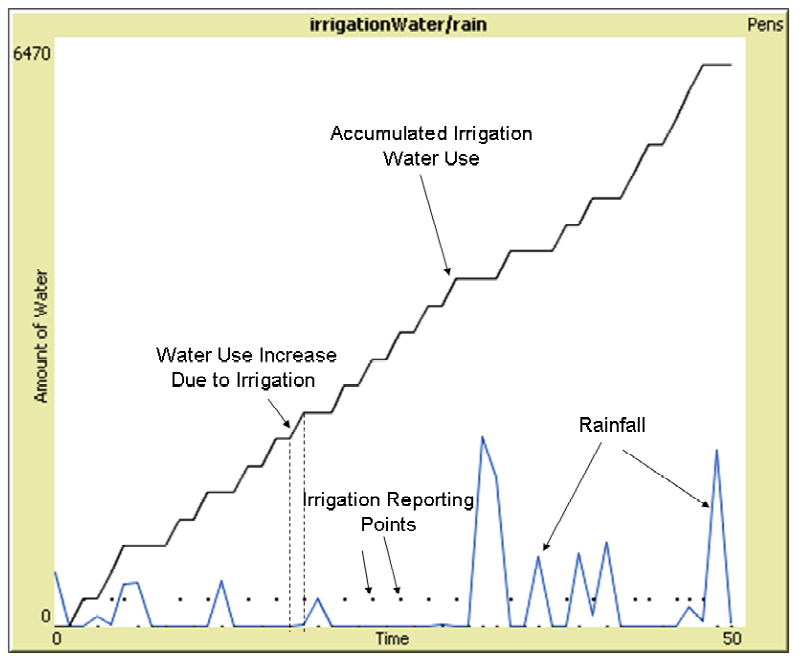

Figure 6: The accumulated irrigation water use for 50 units of time under a uniformly distributed irrigation treatment. Dots are used to report irrigation events in the previous time period.

A deficit irrigation simulation model uses heterogeneous information to make decisions on irrigation treatments (i.e. when and how much to apply). Unlike the point sensor-based uniform irrigation treatment, deficit irrigation scheduling maximises the actual and predicted short-term rainfall events. As shown in Figure 7 , at the initial stage of irrigation, the sprinklers are not triggered as a 
result of balancing the effects of current rainfall and the future probability of rain on dry cubes in the root zone. In some circumstances, the simulation model uses a deficit irrigation treatment ( 0.5 of accumulated ET in this case) since the probability of rainfall is moderate. Applying a reduced amount of irrigation minimises the risk of irreversible damage to plants whilst improving rainfall utilisation. Figure 7 shows an accumulated water consumption of 4520 units over 50 units of time, which represents a water saving of roughly $27 \%$, compared with the point sensor-based irrigation strategy. We recorded 22 irrigation events in this scenario.

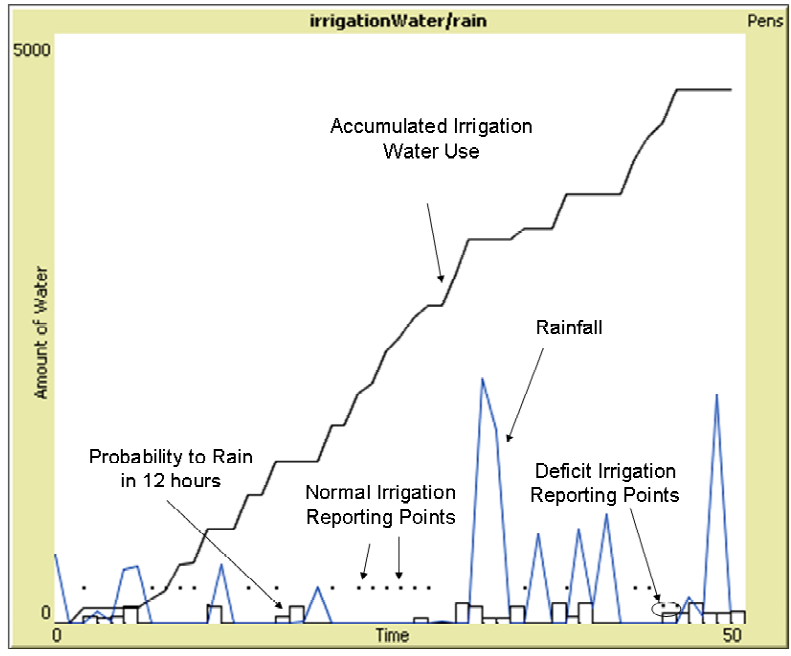

Figure 7: Accumulated irrigation water use in a deficit irrigation scenario.

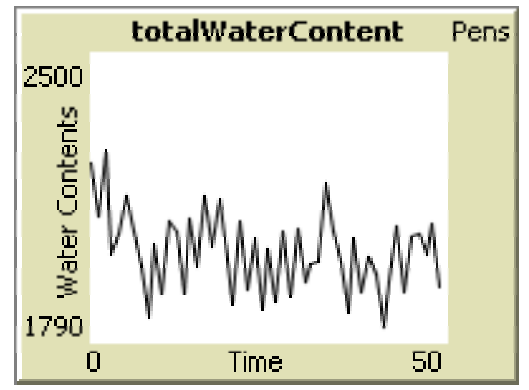

Figure 8: The total water content for point sensor-based uniform irrigation scenario.

In both scenarios, soil water contents are maintained to be below field capacity. As the deficit irrigation model integrates information about future rainfall events, it adapts irrigation frequency based on the predicted contribution 
of rainfall to soil water content. The frequency adaptation which is presented in Figure 7 does not occur under a point sensor-based irrigation scenario. This has a direct impact on the amounts of water consumed. Because the two scenarios are assumed to have identical environmental variables (ET, rainfall, drainage), the difference between the total soil water contents (shown in Figures 8 and 9) is an indication of over-irrigation in the point sensor-based strategy. That is, assuming production is fairly constant above the refill threshold point, which water content is maintained above this level in both scenarios, the extra water applied in the point sensor-based strategy is of no benefit to production.

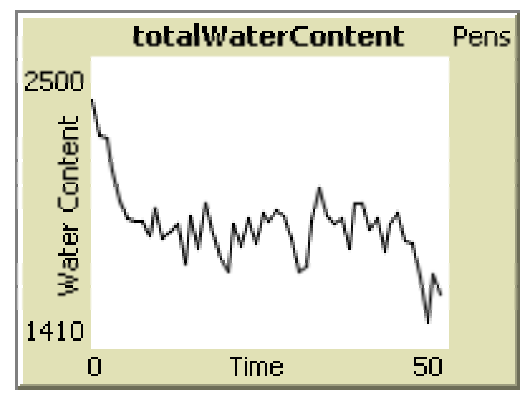

Figure 9: The total water content for the deficit irrigation scenario.

\section{Conclusion}

This paper introduces an information fusion framework to support decisionmaking in deficit irrigation scheduling. The simulation results demonstrate how real-time data collection may be used to improve water use efficiency by considering future rainfall events and practicing targeted irrigation application. The simulation model is however a reduction of a physical phenomenon. To increase the credibility of the model, data obtained from real-time sensors to enhance/calibrate the simulation model is required. At present, the simulation model treats drainage as a constant boundary condition. It is necessary to include beyond root zone soil moisture sensors to approximate deep drainage to further improve the resolution of irrigation decisions.

The intention is to consider crop yield models, multiple-objective optimisation and remote sensing in the next phase of research.

Field quantification is still required to validate this model-data fusion approach for deficit irrigation.

\section{Acknowledgements}

This work is part of a research project jointly funded by the CSIRO Water for a Healthy Country Flagship and the Australian Government through the Intelligent Island Program. The Intelligent Island Program is administered by the Tasmanian Department of Economic Development. We are grateful to all 
members of the CSIRO Tasmanian ICT Centre and the Tasmanian Institute of Agricultural Research for their involvement in this project. Meisha HollowayPhillips also receives project support from Dairy Australia.

\section{References}

[1] ABS, Water Account for Australia 2004 to 2005, cat. no. 4610.0, ABS, Canberra, 2006.

[2] Hennessy, K.B., Fitzharris, B.C., Bates, N., Harvey, S.M., Howden, L., Hughes, J., Salinger, J. \& Warrick, R., Australia and New Zealand (Chapter 11). Climate Change 2007: Impacts, Adaptation and Vulnerability. Contribution of Working Group II to the Fourth Assessment Report of the Intergovernmental Panel on Climate Change, eds. M.L. Parry, O.F. Canziani, J.P. Palutikof, P.J. van der Linden \& C.E. Hanson, Cambridge University Press: Cambridge, pp. 507-540, 2007.

[3] Scheierling, S., Cardon, G. \& Young, R., Impact of irrigation timing on simulated water-crop production functions. Irrigation Science, 18(1), pp. 23-31, 1997.

[4] Costa, M.J., Ortuno, M.F. \& Chaves, M.M., Deficit irrigation as a strategy to save water: physiology and potential application to horticulture. Journal of Integrative Plant Biology, 49(10), pp. 1421-1434, 2007.

[5] Gowing, J.W. \& Ejieji, C.J., Real-time scheduling of supplemental irrigation for potatoes using a decision model and short-term weather forecasts. Agricultural Water Management, 47(2), pp. 137-153, 2001.

[6] Ganji, A., Ponnambalam, K., Khalili, D. \& Karamouz, M., A new stochastic optimisation model for deficit irrigation. Irrigation Science, 25(1), pp. 63-73, 2006.

[7] Bastiaanssen, W.G.M., Allen, R.G., Droogers, P., D’Urso, G. \& Steduto, P. Twenty-five years modelling irrigated and drained soils: State of the Art. Agricultural Water Management, 92(3), pp. 111-125, 2007.

[8] Hall, D.L., McMullen, S.A.H., Mathematical Techniques in Multisensor Data Fusion, Artech House Inc.: Norwood, 2004.

[9] Steinberg, A.N., Bowman, C.L. \& White, F.E., Revisions to the JDL data fusion model. Proc. of the SPIE on Sensor Fusion: Architectures, Algorithms, and Applications, ed. B.V. Dasarathy, pp. 430-441, 1999.

[10] Waltz, E. \& Llinas, J., Multisensor Data Fusion, Artech House: Norwood, 1990.

[11] White, F.E., A Model for Data Fusion. Proc. of the $1_{\text {st National Symposium }}$ on Sensor Fusion, 1988.

[12] Endsley, M.R., Toward a theory of situation awareness in dynamic systems. Human Factors 37(1), pp. 32-64, 1995.

[13] Endsley, M.R., Theoretical underpinnings of situation awareness: A critical review. Situation Awareness Analysis and Measurement, eds. M.R. Endsley \& D.J. Garland, LEA: Mahwah, pp. 3-32, 2000.

[14] Pew, R.W., The State of Situation Awareness Measurement: Heading Toward the Next Century (Chapter 2). Situation Awareness Analysis and 
Measurement, eds. M.R. Endsley, D.J. Garland, Lawrence Erlbaum: Mahwah, pp. 33-47, 2000.

[15] Morgan, K.T., Parsons, L.R. \& Wheaton, T.A., Comparison of laboratoryand field-derived soil water retention curves for a fine sand soil using tensiometric, resistance and capacitance methods. Plant and Soil, 234(2), pp. 53-157, 2001.

[16] van Genuchten, M. Th., A closed-form equation for predicting the hydraulic conductivity of unsaturated soils. Soil Science Society of America Journal, 44 (5), pp. 892-898, 1980.

[17] Šimůnek, J., and M. Th. van Genuchten, Using the HYDRUS-1D and HYDRUS-2D codes for estimating unsaturated soil hydraulic and solute transport parameters. Characterization and Measurement of the Hydraulic Properties of Unsaturated Porous Media, eds. M.Th van Genuchten, F.J. Leij \& L. Wu, University of California: Riverside, pp. 1523-1536, 1999.

[18] Quinlan, J.R., Improved use of continuous attributes in c4.5. Journal of Artificial Intelligence Research, 4, pp. 77-90, 1996.

[19] WEKA, machine learning software package, the University of Waikato, http://www.cs.waikato.ac.nz/ml/weka/.

[20] NetLogo, http://ccl.northwestern.edu/netlogo/ 